\title{
Central nervous system (CNS) involvement in acute leukemia and lymphoma: indications for CNS assessment and advice for diagnostic strategy ${ }^{1)}$
}

\begin{abstract}
Involvement of the central nervous system (CNS) must be considered in leukemia and lymphoma to develop a promising treatment strategy. In particular, a relapse within the CNS compartment is associated with a dismal prognosis. Therefore, CNS involvement should be detected as early as possible during the disease course.
\end{abstract} The risk for CNS disease varies considerably between the diseases. For diseases with a proven high-risk for CNS involvement, such as acute lymphoblastic leukemia (ALL) or Burkitt's lymphoma, prophylactic strategies are used. Although not in every entity, evidence-based therapeutic standards do exist, the detection of tumor cells in the cerebrospinal fluid (CSF) will have an impact on the therapeutic concept. This holds true for the preemptive situation with low tumor burden as well as for the therapeutic situation with a symptomatic patient. Whereas parenchymatous lesions are detected by magnetic resonance imaging, CSF analysis is the method of choice for the diagnosis of neoplastic meningitis. Cytomorphology of CSF cells, if used by an experienced cytopathologist, is of sufficient specificity but limited sensitivity for the diagnosis of leukemia. In lymphoma, cytomorphology has only modest specificity, because rather seldom conclusions can be drawn about the lineage commitment of the lymphoma cells and there is no possibility to prove clonal disease. Furthermore, it is often very difficult to discriminate between neoplastic meningitis and reactive pleocytosis. By combining cytomorphology with flow cytometry, sensitivity as well as specificity can be improved. Careful acquisition of the CSF sample by lumbar puncture is important. Only the last of several CSF fractions should be assessed by cytomorphology and flow cytometry to avoid contamination with peripheral blood. It is further mandatory that essential clinical information is provided by the clinician to the laboratory, so that a well-suited antibody panel can be chosen. This will save material and laboratory time and will further accelerate analysis. Owing to the limited number of cells within the CSF, analysis should always start with a basic antibody panel, which can then be extended on demand.

Keywords: central nervous system; cerebrospinal fluid; leukemia; lymphoma.

\footnotetext{
1) Original German online version at: http://www.degruyter.com/ view/j/labm.2012.36.issue-1/jlm-2011-0039/jlm-2011-0039. $\mathrm{xml}$ ?format=INT. The German article was translated by Compuscript Ltd. and authorized by the authors.

*Correspondence: PD Dr. Mark Ringhoffer, III. Medizinische Klinik, Städtisches Klinikum Karlsruhe, Moltkestr. 90, 76133 Karlsruhe, Deutschland, Tel.: +49-721-9743007, Fax: +49-721-9743049, E-Mail: mark.ringhoffer@klinikum-karlsruhe.de Martin Bommer: Klinik für Innere Medizin III, Universitätsklinikum Ulm, Ulm, Deutschland
}

\section{Introduction}

The diagnosis of involvement of the central nervous system (CNS) plays an important role in hematologic diseases. The two diseases that are most frequently associated with dissemination of malignant cells into the CNS are leukemias and lymphomas. Within these disease entities, the incidences of an infestation of the CNS vary considerably. The indication for CNS diagnosis and the type of diagnosis is therefore dependent on the correct primary diagnosis and the anticipated presentation of the underlying disease. In principle, we distinguish between a presentation as parenchymatous, sometimes even space-occupying lesion, and a manifestation of neoplastic meningitis (MN), in which there is a proliferation of tumor cells in the cerebrospinal fluid (CSF) and the meninges. In this overview, first, the frequency of CNS involvement in various leukemias and 
lymphomas and, derived from this, the indications for CSF diagnostics are to be presented. In the second part, the methodology of the CSF diagnostics is presented and evaluated. The last section derives the implications of the diagnosis and provides information on complementary imaging.

\section{Frequency and type of CNS manifestations in acute leukemias and lymphomas}

\section{Acute leukemias}

The exact incidence of CNS involvement at the time of diagnosis in leukemias and lymphomas is difficult to determine because not all diseases include an examination of the CSF or imaging of the CNS as part of the initial work-up.

In acute myeloid leukemia (AML), older retrospective data from two Eastern Cooperative Oncology Group (ECOG) studies revealed an incidence of 5\% during the entire treatment period, but neither of these studies provided for routine lumbar puncture during the initial diagnosis in the treatment protocol [1]. Consequently, the CSF samples from which an attack on the CNS was diagnosed were generally obtained at the onset of clinical symptoms. Even the data from Brinch et al. [2], which also examined retrospective data over a period of 15 years, revealed a CNS incidence of approximately $5 \%$. Risk factors for developing CNS involvement include a high circulating blast count and, in the univariate analysis, the manifestation of the disease as AML with myelomonocytic or monocytic differentiation [AML FAB M4 or AML FAB M5 according to French American British (FAB) classification]. A retrospective analysis of pediatric leukemias confirmed this for the occurrence of an isolated CNS relapse and also added the existence of a hepatosplenomegaly or 11q23 rearrangement as a risk factor [3]. It is obvious that the nature of the administered chemotherapy, such as the use of CSF-penetrating substances like high-dose cytosine arabinoside, has an important influence on the manifestation of CNS involvement during the course of the disease, which means that one can only speculate as to the true incidence in adults at the time of the initial diagnosis. Own data, which involved subjecting asymptomatic patients with AML before a planned allogeneic stem cell transplantation to a CSF analysis as part of the transplantation preparations, indicate a higher incidence, which was as high as 19\% in refractory patients [4]. Although the current guidelines of the European LeukemiaNet do not require a CNS evaluation in clinically asymptomatic patients [5], this analysis suggests that a CNS evaluation via CSF analysis is useful in certain patient groups.

In ALL, data on the primary CNS involvement are much better, because historical data document a very high rate of CNS manifestations. For this reason, each study protocol now provides for a regular lumbar puncture and intrathecal administration of chemotherapeutic agents. In addition, prophylactic cranial irradiation is included early in the induction phase. This form of combined CNS prophylaxis was able to significantly reduce the incidence of over $50 \%$ of CNS relapses in ALL [6]. The incidence for primary diagnosis is covered by the publication of large study groups, such as GMALL in Germany or GET-LALA in France. These report consistent incidence rates of $7 \%$ at the time of diagnosis and CNS relapses in $15 \%$ of patients properly treated with CNS prophylaxis [7, 8]. For both acute leukemias (AML and ALL), it is true that a CNS recurrence in more than $90 \%$ of patients is followed by a systemic relapse, although a remission in the CNS cannot be achieved in $60 \%$ of patients. In two analyses by the MD Anderson Cancer Center, a B cell karyotype, a value of lactate dehydrogenase $(\mathrm{LDH})>600 \mathrm{U} / \mathrm{L}$, an L3 phenotype of blasts [9], and the coexpression of CD56 [10] were independent risk factors for the occurrence of a CNS relapse. The data presented here relate to neoplastic meningitis; systematic surveys of the occurrence of chloromas (also: myeloid sarcoma, extramedullary myeloid tumor, or granulocytic sarcoma) in the CNS are not found in the literature.

\section{Lymphomas}

The situation with lymphomas is significantly more complex given the large number of subgroups in the classification. In Hodgkin's lymphoma, a CNS involvement is an extremely rare event [11] and there are only a few case reports.

Among non-Hodgkin's lymphomas, one should first distinguish between primary CNS lymphomas (PCNSL), which manifest themselves only in the CNS, and secondary CNS lymphomas in connection with an underlying systemic disease of lymphoma. The PCNSL usually manifest themselves as parenchymal lesions; concomitant meningitis can be detected in $11 \%$ of cases. A special position within the PCNSL is occupied by the primary intraocular 
lymphoma that often affects the vitreous body or uvea and where a concomitant meningitis can be detected in up to $43 \%$ of cases.

Some rare subtypes of non-Hodgkin's lymphomas (NHLs) carry a very high-risk of CNS involvement already at the time of diagnosis. These include histological subtypes of lymphoblastic lymphoma (23\%), Burkitt's lymphoma (24\%), natural killer (NK)/T cell lymphoma of the nasal type (21\%) and HIV-associated lymphomas (25\%).

Much more frequently, however, the clinician will find him or herself confronted with systemic lymphomas as either indolent (low grade) NHL or aggressive (highly malignant) NHL. In low grade NHL, CNS involvement occurs only in very few patients. A retrospective analysis [12] of the Hadassah University Hospital in Jerusalem showed that one must assume an incidence of $<3 \%$. The seven patients in this analysis with CNS involvement were diagnosed with the following: small cell lymphocytic lymphoma ( $\mathrm{n}=2)$, follicular lymphoma (FL) grade I $(\mathrm{n}=2)$, FL grade $2(n=2)$ and unclear low grade histology $(n=1)$. Both parenchymal and leptomeningeal manifestation forms were observed. All patients in this series were symptomatic. There are other smaller series of low grade NHL, such as marginal zone lymphoma, which have found in large retrospective analysis both primary and secondary forms of CNS involvement [13]. Thus, if patients with a history of low grade NHL exhibit neurological symptoms, CNS involvement is to be considered a distinct possibility in terms of differential diagnosis. The mantle cell lymphoma should certainly be considered separately, because, although often classified as low grade, it takes an aggressive clinical course that is treated, also in treatment eligible patients, primarily with high-dose chemotherapy with stem cell replacement. Here, especially in connection with blastoid mantle cell lymphoma, CNS involvement is observed in up to $26 \%$ of patients [14].

The entity that was often discussed in recent years due to potential CNS involvement and therefore possibly indicated prophylaxis is diffuse large cell B-NHL (DLBCL). This is a highly malignant lymphoma. There is different information concerning the incidence for primary diagnosis, because for this entity, too, an evaluation of the CNS via CSF analysis is performed only on all symptomatic patients. The stated numbers on the incidence for primary diagnosis - if we exclude the previously mentioned clinical and histological subtypes - are all under 5\%. There may be a subset of patients with occult, asymptomatic involvement at the time of initial diagnosis, which manifests itself as a CNS relapse in the course of treatment or not until after completion in the absence of CNS-directed therapy modalities.
How high the risk is of a CNS relapse during or after systemic treatment is very well documented, however. In a large retrospective study of patients with DLBCL, where no CNS prophylaxis was applied, the risk of CNS relapse was 4.5\% a year after diagnosis. Risk factors were: higher stage of disease, elevated LDH, more than an extranodal manifestation, and poor performance status $[15,16]$. One of the few sources that provide prospective data on the incidence of CNS relapse in DLBCL is the RICOVER 60 study of the DSHNHL (Deutsche Studiengruppe Hochmaligne Non-Hodgkin-Lymphome: German High Grade Non-Hodgkin's Lymphoma Study Group), where in elderly patients aged $61-80$ years, the value of $6 \times \mathrm{CHOP} 14$ was compared to $8 \times$ CHOP14 with and without rituximab [17]. Patients who had a testicular involvement, an involvement of the bone marrow or a highly cervical or cranial involvement received a CNS prophylaxis with intrathecal methotrexate on days 1 and 5 of cycles 1 and 2. No advantage of the prophylaxis could be identified if the patients were treated with rituximab-containing arms. The 2-year incidence of a CNS relapse was 4.1\% [95\% confidence interval (CI) $=2.3-$ 5.9\%] in the rituximab-containing arms and 6.9\% 95\% $\mathrm{CI}=4.5-9.3 \%)$ in rituximab-free arms $(\mathrm{p}=0.017)$. The multivariate analysis identified $B$ symptoms and the occurrence of multiple extranodal manifestations as risk factors [18]. It was found that $65.5 \%$ of the 58 patients who suffered a CNS relapse exhibited parenchymal involvement in crosssectional diagnostic imaging, whereas only $25.9 \%$ developed meningitis alone. The combination of parenchymal involvement and meningitis was rare, with only 8.6\%.

Other studies identified for CNS relapses found an inverse distribution pattern in the incidence of parenchymal manifestations compared with meningitis: approximately two-thirds of patients had meningitis, one-third had parenchymal lesions; a combined involvement revealed an incidence of $15 \%[12,19,20]$.

\section{Procedures for CSF diagnostics}

\section{Specimen collection and sample quality}

In acute leukemia, the occurrence of a CNS relapse is linked with a very poor prognosis. This shows the need for a reliable diagnosis at an early stage of treatment in order to adjust the therapy at the beginning of a possible CSF involvement. In the neurosurgical setting, it has been demonstrated that the probability of detecting tumor cells in the CSF can depend on the site of CSF extraction (intraventricular extraction vs. lumbar puncture). Nevertheless, 
in clinical practice only the extraction of a CSF sample through a lumbar puncture is common. One should be aware, in case of negative results, however, that lesions that are below the meningeal surface are usually not captured by way of lumbar puncture. In diseases where no neoplastic meningitis is to be expected, usually highresolution imaging is indicated by means of magnetic resonance imaging (MRI).

Prior to the collection of a CSF sample, one must always rule out that increased intracranial pressure exists, because otherwise the result could be a life-threatening complication of an upper or lower incarceration. This can be done by means of an ophthalmological assessment of the papilla (ruling out papilledema) or, even more reliably, by means of cross-sectional imaging, such as a computed tomography (CT) or MRI scan. The CSF sample is then collected, ideally, as atraumatically as possible, using an introducer and a Sprotte needle as part of a lumbar puncture. During the extraction of the CSF, it is important to ensure that the risk of contamination by peripheral blood leukocytes be kept as low as possible. This is especially true of leukemic malignancies, such as acute leukemias, chronic lymphocytic leukemia or the leukemic mantle cell lymphoma. One way to ensure this is the collection of CSF in at least three consecutive fractions. Then, only the last sample is subjected to a morphological or cytological analysis. Whenever possible, the lumbar puncture should be performed by an experienced physician following a previous cytoreduction to avoid contamination by peripheral cells. The determination of the erythrocyte count in the CSF facilitates the identification of samples contaminated by peripheral blood that are to be excluded from any further cytological and flow cytometric analyses.

The quality of the subsequent diagnostic procedures is dependent on a number of factors, such as the amount of CSF obtained, the possibility of repeated analysis, the immediate processing of the obtained sample, and the requirement that often cannot be met of collecting the sample at a site that is as close to a suspected lesion as possible [21]. In clinical practice, the sample volume is generally $<10 \mathrm{~mL}$. Therefore, samples obtained should be handled sparingly and clinical information be passed on to subsequent investigators.

\section{Chemical laboratory (non-cell-based) analyses}

The basic analysis of the CSF involves cell count, determination of $\mathrm{LDH}$, total protein, and glucose (in relation to serum glucose). The latter also serves as the demarcation of reactive or inflammatory changes that can often complicate the interpretation of CSF cytology. Although it is tempting to look at molecules that have contributed to CNS penetration, e.g., matrix metalloproteinases, cathepsins, and chemokines, which may be involved in tumor cell migration, these approaches are currently to be regarded as experimental and subject to research. The significance of the vascular endothelial growth factor (VEGF) in the CSF as a meningitis marker has also been evaluated for some time [22]. Depending on the underlying disease, in special cases, molecular methods, such as PCR may be used to detect a clonal IgVH pattern in lymphomas with a $\mathrm{B}$ cell origin or a distinct $\mathrm{T}$ cell receptor rearrangement in $\mathrm{T}$ cell lymphomas $[21,23]$.

\section{Cell-based analyses}

The sole gold standard for the diagnosis of meningitis has long been light microscope cytomorphology.

For this purpose, the cell number is determined by staining a bit of CSF with trypan blue, followed by counting in a counting chamber. To carry out a morphological analysis under optimal conditions, the cell number is then adjusted and a cytospin preparation created. The corresponding slides are dried and usually stained with a MayGruenwald-Giemsa stain. Then, an assessment is made at the light microscope by an experienced cytopathologist. A normal cell count does not rule out meningitis: several studies report unequivocal evidence for tumor cells with normal cell counts [4]. Therefore, clinical practice to order a morphological examination only from a cell count of $5 / \mu \mathrm{L}$ occasionally leads to loss of information. Morphological analysis is hampered mainly by heterogeneity of the cells. Addition of reactive nucleated cells (normal T cells, ependymal cells, macrophages, etc.) can often be difficult to distinguish from malignant hematopoietic cells. In a study by Hegde et al., which only focused on meningitis with highly malignant lymphomas, the median tumor cell content of the CSF was only $7 \%$ in relation to all analyzed cells [24].

Thus, it is not surprising that sensitivity and specificity of cytomorphology have been judged quite differently in the literature. Although there are only few false-positive findings, some authors assume that, for example, in secondary lymphomas the CSF yields a false-negative in connection with meningitis in up to $70 \%$ of cases [19, 25]. A larger series of autopsies showed that cytology was positive before only in 59\% of cases where a leptomeningeal tumor was detected postmortem [26]. Our own data showed discordance between leukemias and lymphomas. 
Although we achieved in all entities a relatively high sensitivity ( $>80 \%$ with acute leukemias and $>90 \%$ with lymphomas), specificity ranged only from $70 \%$ to $80 \%$, particularly in malignant lymphomas. Identification of leukemic cells was $100 \%$ specific [23]. Above all, demarcation of inflammatory CSF pleocytosis, which occurs not infrequently as a result of infection complications during intensive therapy, shows limitations of the purely morphological analysis. Additional procedures are then necessary.

A method that was established for the diagnosis of leukemias and lymphomas in the past 30 years is flow cytometry. This involves cell suspensions from blood, bone marrow, and other body fluids that are incubated with fluorescently marked antibodies; the expression of specific molecules is measured on the surface of cells. The analysis can detect, by means of multichannel devices, the simultaneous presence of several markers and thus allows the subtyping of leukemias and lymphomas to such extent as cannot be achieved through immunohistochemistry or immunocytology. Owing to a very accurate cell count, it is also possible to distinguish small subpopulations within a cell mixture. In recent years, several studies have been published on CSF diagnostics via flow cytometry. It has been shown in this context that particularly the combination with morphology significantly improves sensitivity and specificity of CSF cell analysis [23, 24]. To allow for optimal processing, the samples should be at the laboratory within a few hours after extraction; after centrifugation they are usually placed in phosphate buffered saline (PBS), incubated with antibodies according to the manufacturer's instructions, and then analyzed in the flow cytometer.

In the choice of the antibody panel used, clinical information is essential. The limited number of analyzable cells makes a precise clinical question of the sender (subtype of NHL or leukemia) indispensable, as well as consideration of previous analyses from peripheral blood or bone marrow that can specify the marker panel. Clinical data should be obtained in the event of insufficient information in any case, because this leads to a resource-saving approach and speeds up the investigation. There are several clinical scenarios: if the underlying disease is known, one can restrict the antibody panel to those surface markers that characterize the underlying disease and thus verify the suspected diagnosis of meningitis. If the underlying disease is not yet diagnosed clearly, a broader marker panel will have to be used. Here it seems sensible to start with a base panel and to then expand it gradually. CD45 expression allows for the differentiation between cells of hematopoietic and nonhematopoietic origin (except in rare erythroid malignancies that may be lacking CD45). CD3 (marker for mature T cells) and CD19 and CD20 (markers for mature B cells) allow for a statement about the lineage of lymphoid cells in the CSF. The combination of the described B cell markers in combination with kappa or lambda light chains as an indication of a light chain restriction confirms the clonality in all key entities belonging to $\mathrm{B}$ cell lymphomas. Addition of markers that occur early in lymphocyte development allows for the detection of B precursor cells, such as with C-ALL. To diagnose $\mathrm{T}$ cell lymphomas, one requires an accurate selection of aberrantly expressed markers, as most $\mathrm{T}$ cell markers, such as CD3, CD4, or CD8 also occur on normal T cells. If hematopoietic markers are missing altogether, the possibility of meningeosis caused by cells of a solid tumor should be considered. An overview of useful markers is shown in Table 1. In most cases, one can distinguish by flow cytometry an inflammatory pleocytosis from a leukemic or lymphomatous meningeosis. Even small subpopulations of malignant cells can be differentiated with careful analysis using flow cytometry. For example, a combined cytomorphologic and flow cytometric analysis in 51 patients with newly diagnosed DLBCL showed a surprisingly high incidence of occult tumor cells in completely asymptomatic patients of 22\% [24]. However, all these patients had clinical risk characteristics that favor a CNS involvement. Our own experience and results show that flow cytometry cannot replace morphology. In particular, with an unclear primary diagnosis, morphological diagnosis remains essential, because non-hematopoietic malignancies may not (yet) be diagnosed by way of flow cytometry in the absence of specific markers. Ideally, both investigations are carried out together and assessed by one investigator.

\begin{tabular}{lllll} 
Myeloid leukemia & \multicolumn{4}{l}{ Lymphoproliferative disease } \\
\cline { 2 - 5 } & B-NHL & T-NHL/NK & c/B-ALL & T-ALL \\
\hline Basic panel & & & & \\
CD45 & CD45 & CD45 & CD45 & CD45 \\
CD3 & CD3 & CD3 & CD3 & CD1a \\
CD19 & CD19 & CD19 & CD19 & CyCD3 \\
CD34 & $\kappa$ & CD4 & CD10 & CD3 \\
CD117 & $\lambda$ & CD7 & Td ${ }^{b}$ & CD4 \\
CD33 & CD10 & CD8 & CD34 & TdT \\
Supplementary panel & & & & \\
MP0 & CD38 & CD1a & $\kappa$ & CD2 \\
CD14 & CD138 & CyCD3 & $\lambda$ & CD7 \\
CD13 & CD5 & CD56 & & CD8 \\
\hline
\end{tabular}

Table 1 Marker profiles as a function of diagnosed or suspected underlying disease.

${ }^{\mathrm{a} N K}$, natural killer cell lymphoma; ${ }^{\mathrm{b}} \mathrm{TdT}$, terminal deoxynucleotidyl transferase. 


\section{Additional diagnostic imaging}

Because CSF diagnosis is only able to detect meningeosis in symptomatic patients or in high-risk patients, it is mandatory to conduct supplementary diagnostic imaging in order to demonstrate parenchymal lesions in the CNS. MRI is the method of choice here. In patients with CNS involvement, FLAIR-weighted sequences and T1-weighted contrast-enhanced images often reveal hyperintense lesions. MRI is also most likely to detect affected cranial nerves and meningeal enhancement as a morphological correlate for meningeosis, but has been shown to be of limited sensitivity with respect to the latter in leukemias and lymphomas [27]. Additional information about the presence of a CNS involvement may be provided in the future by positron emission tomography. The operational capability of the standard tracer 18F-fluorodeoxyglucose (18F-FDG), however, is limited, as healthy brain tissue absorbs FDG to a high degree. Fluorodeoxythmidine (FLT) is an alternative tracer that is available. This pyrimidine analog is incorporated into the nucleus not as a function of the metabolism but only in proliferating cells. Therefore, a much more selective representation of proliferating tumor tissue that is surrounded by metabolically active brain tissue can occur. In a study of 34 patients, in determining the physiological distribution patterns, it has been demonstrated that the FLT tracer did not lead to an accumulation in brain tissue; however, if there was a lymphoma in the CNS, it was possible to distinguish it safely from other brain tissue. The absorption of the FLT tracer in highly malignant lymphomas was significantly higher than in low grade lymphomas, which corresponds to the above enrichment process by incorporation into the DNA of the nucleus [28]. Even in a smaller series of AML patients, the FLT tracer exhibited a selective incorporation into proliferating AML cells and thus allowed for the reliable detection of a meningeosis. In patients with a systemic recurrence, a strong signal was found, as expected, in the bone marrow and spleen due to the high proliferation of leukemia cells located there [29].

\section{Conclusions for clinical application}

Important aspects of this study are:

1. How are the patients stratified with respect to their risk for a CNS involvement, and which groups are subjected to regular checks, for example, by spinal tap?
2. What methods should be applied for CSF diagnostics to achieve the greatest possible sensitivity and specificity?

3. What are the consequences of a positive result?

In malignant lymphomas and acute leukemias, the occurrence of a CNS relapse is linked with a very poor prognosis. This shows up the need for a reliable diagnosis at an early time of treatment in order to adjust the therapy at the beginning of a possible CNS involvement. The frequency of CNS involvement varies widely between AMLs and ALLs. In the case of ALL, the incidence of CNS involvement at the time of diagnosis and during the course of therapy is considerable. Therefore, regular CSF diagnosis and prophylactic therapy are an integral part of all current research protocols. In Germany, too, as part of the GMALL-protocol, a number of systemic and intrathecally applied substances, such as cytosine arabinoside or methotrexate, are used in addition to CNS irradiation. If a CNS involvement is identified in the initial diagnosis of ALL and treated accordingly, the prognosis of the patient most likely will not worsen.

By comparison, AML has a low incidence of CNS involvement. Therefore, none of the current protocols provides for a routine evaluation of CSF or specific CNS prophylaxis. Under European Leukemia Net (ELN) guidelines, a CSF diagnosis is recommended only in symptomatic and possibly a small group of high-risk patients [5]. In contrast to ALL, a CNS prophylaxis is not carried out here. Our own data show that the incidence of AML patients could be higher than previously thought. This may also be because systemic recurrences in recent years have been treated successfully and therefore allowing for more patients in very advanced stages of disease still to be treated curatively, i.e., undergoing allogeneic stem cell transplantation. Therefore, such patients should be examined for an occult CNS involvement beforehand. Given the low sensitivity of CSF cytology, a combination with flow cytometry is certainly indicated here. A positive result will affect the choice of conditioning therapy, but also the further treatment measures. A gold standard of treatment does not exist here: consistent intrathecal therapy, such as irradiation of the neuro-axis, can control leukemia in the CNS. But this does not prevent systemic recurrence and, thus, does not improve the long-term prognosis of patients $[4,30]$. A joint evaluation in large study groups should be undertaken to address this difficult question.

In aggressive non-Hodgkin's lymphomas, risk factors were defined that make a CNS diagnosis and CNS prophylaxis necessary. Very early on, histological subtypes [lymphoblastic lymphoma (LBL), Burkitt's lymphoma] and 
clinical entities (HIV-associated lymphomas, intraocular lymphomas) were identified. In recent years, clinical risk factors were added especially to DLBCLs. These risk factors still have not been uniformly defined and vary by study group. The DSHNHL provides, for example, for a CNS-directed therapy with the addition of two blocks of high-dose methotrexate in elderly patients with an elevated $\mathrm{LDH}$, more than an extranodal involvement, and an ECOG >1 (DSHNHL 2004-1; CHOP-R-esc). This intensified treatment is also administered to all patients with testicular involvement.

The value of additional flow cytometry consists currently, especially in the distinction of reactive from malignant pleocytosis. That the detection of an asymptomatic minimal involvement must entail clinical consequences has been suggested by Hegde and colleagues [24], but this has not yet been sufficiently documented. If at the time of diagnosis, a symptomatic CNS involvement is present, the application of CNS-oriented strategies, as they are used, for example, for primary CNS lymphomas, should be considered. However, the application of these sometimes very intensive protocols is considerably more complex and more toxic over the long-term than standard treatment with R-CHOP. Future studies, therefore, must focus on actually confirming the minimum asymptomatic involvement at the time of diagnosis as the starting point of a CNS relapse. Only then will the application of aggressive CNSdirected therapy be justified for such patients.

As with all leukemic diseases, the detection of monoclonal cells in the CSF should be questioned very critically, because a minimal blood contamination during puncture can mimic the diagnosis of meningeosis. Such "pseudomeningeosis" should never be cause for aggressive treatment, and therefore requires prior confirmation [31].

\section{Conflict of interest statement}

Authors' conflict of interest disclosure: The authors stated that there are no conflicts of interest regarding the publication of this article.

Research funding: None declared.

Employment or leadership: None declared.

Honorarium: None declared.

\section{References}

1. Cassileth PA, Sylvester LS, Bennett JM, Begg CB. High peripheral blast count in adult acute myelogenous leukemia is a primary risk factor for CNS leukemia. J Clin Oncol 1988;6:495-8.

2. Brinch L, Evensen SA, Stavem P. Leukemia in the central nervous system. Acta Med Scand 1988;224:173-8.

3. Johnston DL, Alonzo TA, Gerbing RB, Lange BJ, Woods WG. Risk factors and therapy for isolated central nervous system relapse of pediatric acute myeloid leukemia. J Clin Oncol 2005;23:9172-8.

4. Bommer M, von Harsdorf S, Dohner H, Bunjes D, Ringhoffer M. Neoplastic meningitis in patients with acute myeloid leukemia scheduled for allogeneic hematopoietic stem cell transplantation. Haematologica 2010;95:1969-72.

5. Dohner H, Estey EH, Amadori S, Appelbaum FR, Buchner T, Burnett AK, et al. Diagnosis and management of acute myeloid leukemia in adults: recommendations from an international expert panel, on behalf of the European LeukemiaNet. Blood 2010;115:453-74.

6. Mauer AM. Therapy of acute lymphoblastic leukemia in childhood. Blood 1980;56:1-10.

7. Gokbuget N, Hoelzer D. Meningeosis leukaemica in adult acute lymphoblastic leukaemia. J Neurooncol 1998;38:167-80.

8. Reman O, Pigneux A, Huguet F, Vey N, Delannoy A, Fegueux N, et al. Central nervous system involvement in adult acute lymphoblastic leukemia at diagnosis and/or at first relapse: results from the GET-LALA group. Leuk Res 2008;32:1741-50.

9. Kantarjian HM, Walters RS, Smith TL, Keating MJ, Barlogie B, $\mathrm{McCredie} \mathrm{KB}$, et al. Identification of risk groups for development of central nervous system leukemia in adults with acute lymphocytic leukemia. Blood 1988;72:1784-9.

10. Cortes J. Central nervous system involvement in adult acute lymphocytic leukemia. Hematol Oncol Clin North Am 2001;15:145-62.

11. Gatán L, Sánchez AC, Cantos B, Provencio M. Central nervous system involvement in Hodgkin's lymphoma. Med Oncol 2011;28(Suppl 1):S505-8.

12. Spectre G, Gural A, Amir G, Lossos A, Siegal T, Paltiel O. Central nervous system involvement in indolent lymphomas. Ann Oncol 2005;16:450-4.

13. Bayraktar S, Stefanovic A, Montague N, Davis J, Murray T, Lossos IS. Central nervous system manifestations of marginal zone B-cell lymphoma. Ann Hematol 2010;89:1003-9.

14. Ferrer A, Bosch F, Villamor N, Rozman M, Graus F, Gutierrez $\mathrm{G}$, et al. Central nervous system involvement in mantle cell lymphoma. Ann Oncol 2008;19:135-41.

15. Perez-Soler R, Smith TL, Cabanillas F. Central nervous system prophylaxis with combined intravenous and intrathecal methotrexate in diffuse lymphoma of aggressive histologic type. Cancer 1986;57:971-7.

16. van Besien K, Ha CS, Murphy S, McLaughlin P, Rodriguez A, Amin $\mathrm{K}$, et al. Risk factors, treatment, and outcome of central nervous system recurrence in adults with intermediate-grade and immunoblastic lymphoma. Blood 1998;91:1178-84.

17. Pfreundschuh M, Schubert J, Ziepert M, Schmits R, Mohren M, Lengfelder $\mathrm{E}$, et al. Six versus eight cycles of bi-weekly CHOP-14 with or without rituximab in elderly patients with aggressive 
CD20+ B-cell lymphomas: a randomised controlled trial (RICOVER-60). Lancet Oncol 2008;9:105-16.

18. Boehme V, Schmitz N, Zeynalova S, Loeffler M, Pfreundschuh M. CNS events in elderly patients with aggressive lymphoma treated with modern chemotherapy (CHOP-14) with or without rituximab: an analysis of patients treated in the RICOVER-60 trial of the German High-Grade Non-Hodgkin Lymphoma Study Group (DSHNHL). Blood 2009;113: 3896-902.

19. Ferreri AJ, Assanelli A, Crocchiolo R, Ciceri F. Central nervous system dissemination in immunocompetent patients with aggressive lymphomas: incidence, risk factors and therapeutic options. Hematol Oncol 2009;27:61-70.

20. Hollender A, Kvaloy S, Lote K, Nome O, Holte H. Prognostic factors in 140 adult patients with non-Hodgkin's lymphoma with systemic central nervous system (CNS) involvement. A single centre analysis. Eur J Cancer 2000;36:1762-8.

21. Chamberlain MC, Glantz M, Groves MD, Wilson WH. Diagnostic tools for neoplastic meningitis: detecting disease, identifying patient risk, and determining benefit of treatment. Semin Oncol 2009;36:S35-45.

22. Stockhammer G, Poewe W, Burgstaller S, Deisenhammer F, Muigg A, Kiechl S, et al. Vascular endothelial growth factor in CSF: a biological marker for carcinomatous meningitis. Neurology 2000;54:1670-6.

23. Bommer M, Nagy A, Schopflin C, Pauls S, Ringhoffer M, Schmid $M$. Cerebrospinal fluid pleocytosis: pitfalls and benefits of combined analysis using cytomorphology and flow cytometry. Cancer Cytopathol 2011;119:20-6.
24. Hegde U, Filie A, Little RF, Janik JE, Grant N, Steinberg SM, et al. High incidence of occult leptomeningeal disease detected by flow cytometry in newly diagnosed aggressive B-cell lymphomas at risk for central nervous system involvement: the role of flow cytometry versus cytology. Blood 2005;105:496-502.

25. Yoshida S, Morii K, Watanabe M, Saito T. Characteristic features of malignant lymphoma with central nervous system involvement. Surg Neurol 2000;53:163-7.

26. Glass JP, Melamed M, Chernik NL, Posner JB. Malignant cells in cerebrospinal fluid (CSF): the meaning of a positive CSF cytology. Neurology 1979;29:1369-75.

27. Pauls S, Fischer AC, Brambs HJ, Fetscher S, Hoche W, Bommer $M$. Use of magnetic resonance imaging to detect neoplastic meningitis: limited use in leukemia and lymphoma but convincing results in solid tumors. Eur J Radiol 2012;81:974-8.

28. Buck AK, Bommer M, Stilgenbauer S, Juweid M, Glatting G, Schirrmeister $\mathrm{H}$, et al. Molecular imaging of proliferation in malignant lymphoma. Cancer Res 2006;66:11055-61.

29. Buck AK, Bommer M, Juweid ME, Glatting G, Stilgenbauer S, Mottaghy FM, et al. First demonstration of leukemia imaging with the proliferation marker $18 \mathrm{~F}$-fluorodeoxythymidine. J Nucl Med 2008;49:1756-62.

30. Sanders KE, Ha CS, Cortes-Franco JE, Koller CA, Kantarjian HM, Cox JD. The role of craniospinal irradiation in adults with a central nervous system recurrence of leukemia. Cancer 2004;100:2176-80.

31. Nowakowski GS, Call TG, Morice WG, Kurtin PJ, Cook RJ, Zent CS. Clinical significance of monoclonal B 4cells in cerebrospinal fluid. Cytometry B Clin Cytom 2005;63:23-7. 